\title{
Der Ort der Verurteilung Jesu.
}

\author{
Von J. Kreyenbühl in $Z$ ürich.
}

Über den Ort, wo Pilatus das Urteil über Jesus gesprochen hat, scheinen immer noch $Z$ weifel und Zweideutigkeiten zu walten. Mc erzählt 15, I6-20 die Verspottung des Verurteilten durch die römischen Soldaten, welche ihn in das Innere des Hofes, welcher das Prätorium ist, abführten und dort mit ihm ihre rohen Spässe trieben. In dieser Angabe ist vorausgesetzt, dass die Verurteilung Jesu zum Tode und die sofortige Geisselung ebenfalls im Prätorium und zwar in äusseren Teile, auf dem Vorplatze, wo der Richterstuhl stand, stattgefunden hat. Es ist nicht notwendig anzunehmen, dass auch die Geisselung im Innern des Hofes, welcher das Prätorium ist, vorgenommen worden sei. Denn sowohl bei Mc als bei Mt schliesst sich unmittelbar an das Todesurteil der Befehl zur Geisselung an und erst nach dem Vollzug dieser Strafe übergiebt Pilatus Jesus den Soldaten zur Kreuzigung (Mt 27, 26. Mc 15, 15). Auch die Angabe, dass die Soldaten Jesus erst mit dem roten Soldatenmantel bekleideten und ihm nachher seine eigenen Kleider wieder anzogen, beweist, dass Jesus vorher, eben zur Geisselung, entkleidet und so in das Innere der aủhń abgeführt worden ist. Auch Gessius Florus lässt vornehme Juden vor seinem Richterstuhle geisseln und darauf (doch wohl nicht an derselben Stelle) ans Kreuz schlagen '(Jos. b. j. II, I4, 9).

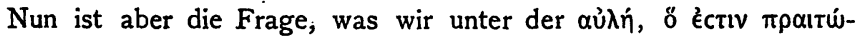
piov zu verstehen haben. Holtzmann neigt sich (Handcomm. I 3 179) zu der Ansicht, es sei die Kaserne der Burg Antonia gemeint. „Dort im Wachtzimmer, versammeln die Soldaten die ganze Kohorte, oder falls die Zahl von 600 Soldaten zu hoch erscheinen sollte, eine der Manipeln; deren drei auf eine Kohorte gingen: der Sprachgebrauch lässt beides zu. Gemeint ist die Leibwache des Prokurators." Damit wäre auch gegeben, dass die Verurtẹilung Jesu in der Antonia stattgefunden 
hat. Denn es deutet in den evangelischen Berichten nichts darauf hin, dass zwischen der Verurteilung einerseits und der Geisselung, der Verspottung und der Hinausführung zur Execution eine Ortsveränderung stattgefunden habe. Nur darin ist Mc genauer als Mt, dass jener dic Verspottung des sciner Kleider beraubten und gegeisselten Königs der Juden ěcw rịc aủhîc vor sich gehen lässt, während dieser überhaupt vom Prïtorium redet. Aber selbstverständlich gehört auch der äussere Teil, wo nach der ganzen Schilderung der Synoptiker die Verhandlung des Pilatus mit den Hierarchen, der Tumult der Volksmassen, das Verhör mit Jesu, die Fireigebung des Barabbas und die Verurteilung Jesu

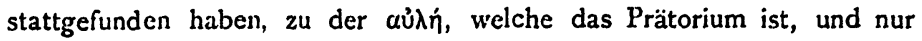
eben dic Verspottungsscenen und die unmittelbaren Vorbereitungen zur Kreuzigung haben, weil sie bloss von den römischen Soldaten ausgingen,

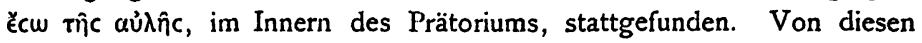
innern Lokalen aus ist Jesu zur Kreuzigung hinausgeführt worden. Das Prätorium also ist in seinen äusseren und innem Räumlichkeiten der Ort, wo Pilatus das Urteil gesprochen und Jesus die Geisselung und Verspottung erduldet hat. Der Ansicht Holtzmanns aber, dass unter dem Prätorium die Kaserne der Burg Antonia zu verstehen sein möchte, stehen die sehr bestimmten Angaben des Josephus entgegen, wonach die römischen Procuratoren, wenn sie in. Jerusalem sich aufhielten, den Palast des Königs Herodes in der Oberstadt zu ihrem Aufenthaltsorte wählten. So bemächtigt sich schon während der Aufstände unter dem Ethnarchen Archelaus der Procurator von Syrien, Sabinus, des Herodespalastes in Jerusalem (Jos., b. j. II, 2, 2; Ant. XVII, 9, 3). Von Gessius Florus, dem letzten Statthalter Judäas vor dem grossen Kriege (64-66), berichtet Josephus an der angezogenen Stelle (b. j. II, I4, 8) ausdrücklich, dass er im Königspalaste Wohnung genommen habe. Vor demselben ( $\pi \rho$ ò aủtŵv), d. h. vor dem eigentlichen Palaste, auf einem freien Platze, stellte er seinen Richterstuhl auf und liess die Mächtigen und Vornehmen der Stadt und die Hohenpriester vor sich kommen, damit sie sich verantworteten. Von der Antwort der Notabeln nicht befriedigt, erteilt der Procurator den Soldaten Befehl zu Plünderung und Mord und lässt eine Menge Bewohner, darunter solche ritterlichen Standes, geisseln und ans Kreuz schlagen. Hier haben wir also eine Scene vor uns, die in allen Einzelheiten der Verurteilung Jesu durch Pilatus entspricht. Daraus dürfen wir mit Recht -schliessen, dass bei der Verurteilung Jesu auch die Örtlichkeit dieselbe gewesen ist, d. h., dass sie nicht in der Antonia, sondern auf dem grossen freien Platze vor dem Herodespalaste statt- 
gefunden hat. Hier ist also, wie auch Spiess (Das Jerusalem des Josephus S. 23), Grimm (s. v. זpaıtúpıov) und neuerdings Guthe (Hauck, R. E. 8,686 ) urteilen, das Prätorium zu suchen.

Zur Bestätigung dieser Auffassung dient eine bis jetzt in dieser Richtung noch nicht verwertete Nachricht des Josephus, welche den Procurator Pontius Pilatus selbst betrifft. Wie der jüdische Geschichtsschreiber an zwei Stellen (b. j. II, 9, 4, ant. XVIII, 3, 2) erzählt, erregte Pilatus dadurch den heftigen Unwillen der Juden, dass er die Kosten für die Anlage einer grossen Wasserleitung nach Jerusalem aus dem Tempelschatze bestritt. Da nun Pilatus sich in Jerusalem aufhielt, so umstand die Volksmenge den auf dem Richterstuhl sitzenden Procurator und gab ihren Unwillen durch Lärmen und Toben zu erkennen. Aber Pilatus hatte den nahënden Sturm vorausgesehen und daraufhin Massregeln getroffen, die seiner ebenso listigen wie gewaltthätigen Natur entsprachen. Er gab den bewaffneten Soldaten Befehl, sich in bürgerlicher Kleidung unter die Menge zu mischen, verbot ihnen den Gebrauch des Schwertes, bewaffnete sie aber mit Knütteln und gab dann vom Richterstuhle aus das Zeichen, mit den Stöcken über die schreiende Menge herzufallen. Das geschah, viele Juden kamen durch die Schläge um, andere wurden auf der Flucht und in der allgemeinen Verwirrung durch die eigenen Volksgenossen zu Boden getreten und getötet, der Aufstand aber ohne eigentliches Blutvergiessen ersticht.

Nun ist aber ganz klar, dass dieser Tumult sich nur vor dem Herodespalaste abgespielt haben kann, auch wenn Josephus dieser Örtlichkeit hier nicht ausdrücklich Erwähnung thut. In der Antonia selbst kann sich dieser Aufruhr nicht ereignet haben, denn die Volksmassen wären von den römischen Soldaten sicher nicht in die Burg eingelassen worden. Auf den Tempelplatz aber unterhalb der Antonia, zu welchem Stufen hinabführten, wird niemand das $\beta \hat{\eta} \mu \alpha$ des Landpflegers verlegen wollen. Vielmehr wird das Tribunal genau da gestanden haben, wo es unter Gessius Florus gestanden hat.

Man könnte nun freilich auf den Aufstand hinweisen, den die letzte Anwesenheit des Paulus in Jerusalem (Act 21-23) erregt hat. Dieser hat sich allerdings zwischen dem Tempelplatze und der Tempelburg abgespielt und Paulus ist vom Tribunen der Cohorte in die Kaserne der Antonia in Sicherheit gebracht worden (Act 21, 34. 37; 22, 24; 23, 10. 16. 32). Allein hier tiandelt es sich eben nicht um die Anwesenheit des Procurators, der zu dieser Zeit in Cäsarea war, und der Schauplatz ist ganz unzweideutig durch den Tempel, die $\pi \alpha \rho \in \mu \beta 0 \lambda \eta ́ n$ und die 


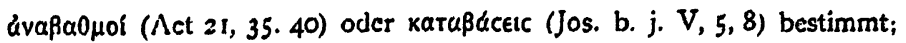
dagegen ist der von Joscphus erzählte Aufruhr unmittelbar gegen den Procurntor gerichtet, der in seiner amtlichen Stellung angegriffen wird.

Was dic Anwescnheit der Soldaten anbetriff, welche in den synoptischen Berichten bei der Verurteilung Jesu überall in der Gegenwart des Procurators vorausgesctzt werden, so ist ganz selbstverständlich, dass nicht nur dic Antonia cine Besatzung gehabt hat, sondern auch der Herodespalast, und dass der Procurator bei seiner Anwesenheit im Palaste allermindestens durch eine Leibwache geschützt gewesen ist. Wenn Mc 15, 16 erzählt, die Soldaten hätten bei der Verspottung Jesu die "ganze Cohorte" zusammengerufen, so kann darunter auf keinen Fall die Besatzung der Burg Antonia, 600 Mann, verstanden sein, sondern es ist die Besatzung des Herodespalastes oder ein Teil derselben, die Leibwache des Procurators, gemeint. Daher ist auch im Berichte der Synoptiker über die Execution des Todesurteils nirgends vom $\chi^{\imath} \lambda i \alpha^{2} \rho \chi 0 c$

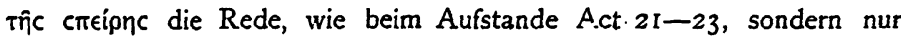
vom kevtupíwv (Mc 15,39), der die zum Vollzug des Todesurteils beorderten Soldaten befehligt hat. Die Angabe Joh 18, 3. I2 ist schon deshalb ungeschichtlich. Es wäre ja in der That lächerlich gewesen, eine ganze Cohorte gegen einen Einzigen aufzubieten und dazu noch Diener der Hohenpriester und Pharisäer mit Fackeln und Lampen und Waffen in Bewegung zu setzen. Das Aufgebot einer solchen Truppenmacht hätte auch schlecht gestimmt zu der Heimlichkeit, mit welcher die Hierarchen unter Benutzung der Stille und des Dunkels der Nacht und der Tücke des Verrates die Gefangennahme Jesu ins Werk gesetzt haben. Gedachte man doch zuerst überhaupt mit Rücksicht auf einen während des Festes zu fürchtenden Volksauflauf die Verhaftung Jesu bis auf die Zeit nach dem Feste $z u$ verschieben (Mc 14, 2) und wurde man erst durch den Verrat des Judas zu einer früheren Ausführung des Planes bewogen (Mc 14, I0). Die Gefangennahme Jesu aber war durchaus und ausschliesslich das Werk der Synedristen und ihres Werkzeuges, des Verräters, und die römischen Soldaten traten erst in Thätigkeit, als das Urteil zu vollziehen war. „Nur bei der Verhöhnung und Hinrichtung des sçhon der römischen Macht Übergebenen sind Legionarsoldaten am Platz. Vorher wären diese den Juden nur mit Bewilligung des Procurators zu Gebote gestanden, und am wenigsten wäre bei solcher Verhaftung der tópußoc Mc 14, 2=Mt 26, 5 zu vermeiden gewesen" (Holtzmann, Handcomm. IV, 185). Die Angabe Mc 14, 43 ist daher einzig correct. Von der Teilnahme der Besatzung der Antonia an der Ver- 
haftung Jesu ist also gänzlich abzusehen und die Beteiligung römischen Militärs bei den Vorbereitungen zur Hinrichtung Jesu und bei dieser selbst ist durch die Örtlichkeit, den Herodespalast, und die Anwesenheit des Procurators in demselben, ohne Weiteres gegeben. War doch der Palast zugleich die feste $Z$ wingburg der Oberstadt, die als solche geradezu neben die Antonia gestellt wird, welche die gleiche Bedeutung für den Tempel hatte (Jos. ant. XV, 7.8); daher wird der Palast von

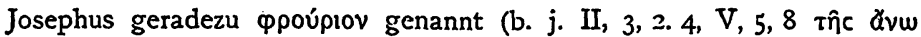

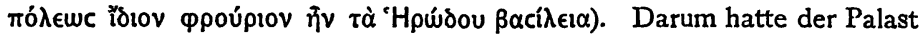
mit seinen drei grossen Türmen auch eine eigene, in kriegerischen Zeiten gewiss bedeutende, Besatzung, welche während der jüdischen Aufstände wiederholt in Action trat (b. j. II, 2, 2; 3, 1. 2; 17, 6. 8). Vielleicht liegt gerade in dieser Thatsache, dass der Palast des Procurators eine militärische Besatzung hatte, der Grund für die Benennung der $\alpha u ̉ \lambda \dot{~ a l s ~ \pi \rho \alpha ı t u ́ p ı o v . ~ D i e s e r ~ A u s d r u c k ~ b e d e u t e t ~ b e k a n n t l i c h ~ z u n a ̈ c h s t ~}$ das Zelt des Oberbefehlshabers im römischen Lager, sodann den Palast, den der Präses oder Procurator einer Provinz bewohnte, welcher stets zugleich eine kleinere oder grössere Truppenmacht zu befehligen hatte. So heisst der'Palast des Verres in Syrakus, der früher dem König Hiero gehörte, domus praetoria (Cic. Verr. II, 5, 12, 30), so wohnt der Pro-

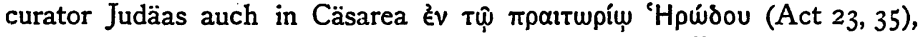
in dem Palaste, den Herodes für sich erbaut hatte. Überall finden wir aber auch den Procurator Judäas als Befehlshaber der Truppen, der Kaıcapeîc und $\Sigma \in \beta \alpha c t \eta v o i$, die aus Einheimischen rekrutiert werden. Auch in Cäsarea ist Pilatus von Truppen umgeben (Jos. b. j. II, 9, 2.3). Die Wohnung des Procurators als Truppenchefs kann darum nach römischem Sprachgebrauch mit Recht als Prätorium bezeichnet werden.

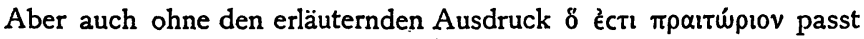
die Bezeichnung $\alpha u ̉ \lambda \eta ́ n$ nicht auf die Antonia, die eine befestigte Burg war. Allerdings hatte das Innere der Antonia den Raum und die Anlage eines Palastes; Gemächer der verschiedensten Art, Säulenhallen, Bäder und geräumige Lagerplätze für Soldaten waren vorhanden, so dass sie Josephus, weil sie alles Erforderliche darbot, mit einer Stadt, wegen ihrer Pracht mit einem Königsschlosse vergleicht (b. j. V, 5, 8). Aber ihre Hauptbestimmung war doch, als Festung $z u$ dienen und bei drohenden Aufständen den Tempelplatz rasch durch Militär zu besetzen, sie war, wie Josephus bündig sagt, das eigens für den Tempel bestimmte ppoúprov, wie der Tempel selbst wieder als ein ppoúpıov für die Stadt gelten konnte. Ihr Hauptbestandteil war daher die Kaserne. (Act 21, 37 
u. s. w.) und ihre Bezcichnung lautet bei Josephus regelmässig nicht

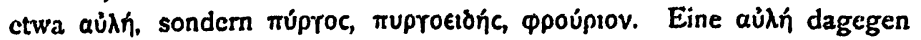
ist der Palast des Hohenpriesters (Mc 14, 54) oder des Kaiscrs (Baci ikn் aủ̇ń Iren. bei Eus. h. c. V, 20, 5). Von Josephus wird (b. j. V, 5, 4)

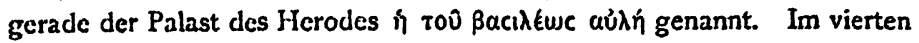
Evangelium (10, 1. 16) heisst der Schafstall so, wobci die ursprüngliche Bedeutung als umfriedigter Ort hervortritt (vgl. Io, I \&vaßaivwv $\& \lambda \lambda \alpha-$ $\chi 0 ́ \theta \in v)$. Nur ist $z u$ beachten, dass in den Gleichnisreden Joh $10,1-16$ Hirte und Schafstall und Thüre des Schafstalles bildliche Bezeichnungen religiöser Gemeinschaften und ihrer Leiter sind, wie namentlich aus v. 16 deutlich hervorgeht. Die $\alpha u ̉ \lambda$ bezeichnet daher im eigentlichen Sinne die betreffende religiöse Gemeinschaft, deren Leiter, guter oder böser, der $\pi 0 \mu \mu \eta \dot{v}$ ist.

Gegen die Beziehung von $\pi p a$ atúptov auf den Herodespalast hat man Lc 23, 7 geltend gemacht, indem man aus dem Ausdrucke àvé$\pi \epsilon \mu \psi \epsilon v$ schloss, dass der Palast des. Herodes Antipas höher gelegen habe, als der Aufenthaltsort des Pilatus. In diesem Falle könnte Pilatus freilich nicht im Palaste des ersten Herodes gewohnt haben, da die Herodier während ihres Aufenthaltes in Jerusalem in dem tiefer nach dem Tyropöon zu gelegenen Palaste der Hasmonäer zu wohnen pflegten

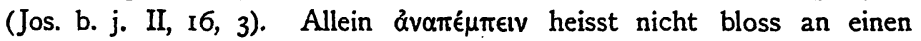
räumlich höher gelegenen Ort schicken, sondern auch an eine höhere Amtsstelle (Grimm s. v.) und wird daher z. B. vom Transporte Gefangener nach Rom gebraucht (Act 25, 21, Holtzmann, Handcomm. I, I, 417; 2, I44). Lc 23, II. I5 hat es die Bedeutung von "zurücksenden“. Es ist also aus diesem Worte kein Anhaltspunkt dafür zu gewinnen, dass Pilatus in der Antonia und nicht in dem höher gelegenen Palaste des Herodes residiert habe.

In allen diesen Ausführungen haben wir absichtlich die Angaben des vierten Evangeliums (18, 28. 33; 19, 9) ausser Betracht gelassen, weil nach unserer Auffassung in allen diesen Angaben, in der ganzen Schilderung der Verhandlungen vor Pilatus u. s. w: keine Geschichte Jesu vorliegt, sondern die verhüllende und allegorisierende Composition des Verfassers auch hier wie in der ganzen Kreuzigungs- und Auferstehungsgeschichte ihre üppigen Blüten trẹibt. Ausführlich ist dies an einer andern Stelle zu zeigen, wenn die Herren Kritiker gestatten, den "verwegenen" Versuch zu Ende zu führen. Aber auch eine wörtliche und geschichtliche Auffassung des "johanneischen" Berichtes würde gleichfalls mit der Annahme unverträglich sein, dass Pilatus die Ver- 
handlungen mit den Hierarchen und Synedristen in der Antonia geführt und dort auch das Todesurteil gesprochen habe. Abgesehen davon, dass es der Würde und auch dem aus der Geschichte bekannten Charakter des römischen Statthalters ganz unangemessen war, zwischen der Antonia und dem Tempelplatze hin und her zu gehen, im Innern des Prätoriums $\mathrm{zu}$ verschwinden und wieder zu der aufgeregten Versammlung hinauszugehen, wie dies Pilatus nach dem Berichte des Vierten gethan haben soll, so hat auch die Bemerkung 18, 28 keinen Sinn, wenn unter dem Prätorium die Antonia gemeint sein soll. War doch der Eintritt in die Burg den Juden und namentlich so aufgeregten Volksmassen, wie sie bei der Verurteilung Jesu erscheinen, gänzlich verschlossen; es stand ihnen gar nicht frei, die Antonia zu betreten, und die Furcht, sich dadurch zu verunreinigen, war völlig unbegründet. Auch Act 21, 27 ff. erscheint das Volk nur auf dem Tempelplatze und Paulus redet $\mathrm{zu} \mathrm{ihm}$ von den Stufen aus $(21,40)$ und wird dann in die

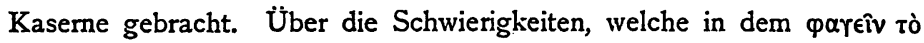
$\pi \alpha ́ c \chi \alpha$ und somit in der Verschiedenheit des synoptischen und johanneischen Berichtes über'den Todestag Jesu liegen, wollen wir gar nicht reden. Aber wie können die Juden das Betreten des Prätoriums aus Frucht vor levitischer Verunreinigung vermieden. haben, wenn sie doch nach den Synoptikern unmittelbar mit Pilatus, also im Prätorium selbst, über den Tod Jesu und die. Freigebung des Barabbas verhandeln? Haben sie sich auch zu verunreinigen gefürchtet, als sie den Pilatus in der Ángelegenheit des. Tempelschatzes bestürmten, als sie vor Gessius Florus erschienen, als sie in der Streitsache des Paulus vor dem Procurator Felix im Prätorium des Herodes in Cäsarea auftraten (Act. 24)? Gewiss haben im Umgange von Juden und Heiden und namentlich in Bezug auf die Tischgemeinschaft Schranken bestanden (Act IO, 28; Gal 2, 12 f.), aber gerade die Angabe des vierten Evangeliums, die Juden hätten aus Furcht vor Verunreinigung das Prätorium des Pilatus nicht betreten wollen, wird durch die ganze Geschichte und durch die Synoptiker selbst widerlegt. Diese Angabe gehört daher keineswegs zu den Beweisen für die Bekanntschaft des Evangelisten mit den Lebensbedingungen und Lebensgewohnheiten des jüdischen Volkes (O. Holtzmann das Joh. Ev. S. I88-191), sie gehört aber auch nicht zu den "offenbaren Missgriffen" die er trotz dieser Vertrautheit begangen haben soll (a. a. O. S. 191-193), sondern sie gehört in das grosse Reich der geheimnisvollen Allegoristik, welches der Enthüllung harrt.

Wir haben darum doch wohl mit Recht auf die Verwendung der 
„johanneischen" Angaben in unserer Frage verzichtet. Wir bedürfen ihrer auch nicht, denn der Beweis ist aus den übrigen Instanzen erbracht, dass Jesus von Pilatus im Palaste des Herodes verhört, verurteilt und von hier aus zum Tode geführt worden ist. Dieses Ergebnis mag ja an sich cine Klcinigkeit sein, aber schliesslich darf nichts eine Kleinigkeit heissen, was einen sicheren und unzweifelhaften Beitrag zur Geschichtc Jesu bedeutet. Auch die alte Kirche hat es nicht für eine Kleinigkeit crachtet, als sie den Pontius Pilatus in ihr Glaubensbekenntnis aufnahm. 